\title{
Correction: Single-cell genomics shedding light on marine Thaumarchaeota diversification
}

\author{
Haiwei Luo • Bradley B Tolar · Brandon K Swan · Chuanlun L Zhang • Ramunas Stepanauskas • Mary Ann Moran • \\ James T Hollibaugh
}

Published online: 20 November 2019

(c) International Society for Microbial Ecology 2019

\section{Correction to: The ISME Journal}

$$
\text { https://doi.org/10.1038/ismej.2013.202 }
$$

Since publication of the original article, the authors have realised some results were inaccurate due to contamination. The authors state:

"The genes encoding catalase we reported in Thaumarchaea single-cell amplified genomes (SAGs) AB-661-
L21 and AB-661-M19 have been determined to be due to contamination of the original SAG sequences. This gene was identified in the early version of the assemblies, but is no longer present in newer versions of the assemblies (Table 1). To the best of our knowledge at the time the MS was completed, there was no evidence that marine Thaumarchaeota contain genes encoding catalase."

The authors apologise for any inconvenience caused.
Table 1 Three versions of the thaumarchaeotal SAGs downloaded from the IMG database (https://genome.jgi. doe.gov/portal/)

\begin{tabular}{lllll}
\hline SAG ID & GBK file in JGI & IMG taxon ID & Submission date & Catalase gene \\
\hline AB-661-L21 & 14021.assembled.gbk & 2524023084 & $2013-05-04$ & Present \\
AB-661-L21 & 40618.assembled.gbk & 2524023084 & $2014-06-16$ & Present \\
AB-661-L21 & 77355.assembled.gbk & 2648501535 & $2016-01-09$ & Absent \\
AB-661-M19 & 14053.assembled.gbk & 2524023095 & $2013-05-04$ & Present \\
AB-661-M19 & 40619.assembled.gbk & 2524023095 & $2014-06-16$ & Absent \\
AB-661-M19 & 77356.assembled.gbk & 2648501536 & $2016-01-09$ & Absent \\
\hline
\end{tabular}

